\section{FREQUENCIA DA PRENHEZ NOS CORNOS UTERINOS E SUA RELAÇĀO COM O SEXO DO PRODUTO EM FËMEAS BOVINAS DA RAÇA HOLANDESA}

WASHINGTON FOGLI SILVEIRA
Médico Veterinário
Instituto de Pesca

Secretaria da Agricultura e Abastecimento de São Paulo

JOSÉ MARQUES REIS

Médico Veterinário

Instituto de Pesca

Secretaria da Agricultura e Abastecimento de São Paulo

OZIEL BIZUTTI

Professor Adjunto

Faculdade de Medicina Veterinária e

Zootecnia da USP

LUIZ BENITO GAMBINI

Médico Veterinário

Instituto de Zootecnia da Secretaria de Agricultura do Estado de São Paulo

\section{CARLOS OLIMPIO MEIRELLES DOS SANTOS} Médico Veterinário

SILVEIRA, W.F.; REIS, J.M.; BIZUTTI, O.; GAMBINI, L.B.; SANTOS, C.O.M. Frequência da prenhez nos cornos uterinos e sua relação com o sexo do produto em fèmeas bovinas da raça Holandesa. Rev.Fac.Med.vet.Zootec.Univ.S.Paulo, 22(1): $59-64,1985$.

RESUMO: No presente estudo verificou-se a alternância de gestaçāo e um possivel efeito do corno uterino prenhe sobre o sexo do produto. De 1.275 fêmeas bovinas da raça Holandesa,variedades malhada de preto e malhada de vermelho, puras de origem e puras por cruzamento, que tiveram suas gestaçōes diagnosticadas através da palpaçāo retal, foram analisados 1.009 partos simples. A análise estatística dos dados consitiu na aplicação do teste de qui-quadrado $\left(\mathrm{X}^{2}\right)$, tendo sido estabelecido o nível de $5 \%$ para a rejeiçāo ou nāo da hipótese de nulidade, verificando-se: - maior frequência de gestaçס̄es no como uterino direito em relação ao corno uterino esquerdo, nas fêmeas bovinas da raça Holandesa variedade maihada de preto, tanto puras de origem como puras por cruzamento, o mesmo não ocorrendo na variedade malhada de vermelho, tanto puras de origem como puras por cruzamento; - tanto na variedade malhada de preto como na variedade malhada de vermelho nāo houve significância entre o sexo do bezerro e o corno uterino onde o mesmo foi gerado.

\section{LITERATURA}

Os clássicos da literatura sobre reprodução animal afirmam que, na espécie bovina, cerca de $60,00 \%$ das ovulações ocorrem no ovário direito e $40,00 \%$ no esquerdo (SALISBURY \& VENDEMARK ${ }^{15}$, ROBERTS ${ }^{14}$, ARTHUR $^{2}$, HAFEZ ${ }^{10}$ ).

$\mathrm{CLARK}^{4}$, trabalhando com cinco raças leiteiras, encontrou através da palpaçฐ̃o retal em 704 gestaçōes, 293 $(41,62 \%)$ localizadas no corno uterino esquerdo e 411 $(58,38 \%)$ localizadas no como uterino direito. Dentre 560 partos, $292(52,14 \%)$ eram machos, $121(41,44 \%)$, gestados no corno esquerdo e $171(58,56 \%)$ no corno direito; 268 $(47,86 \%)$ eram fêmeas, $107(39,93 \%)$ gestadas no corno esquerdo e $161(60,07 \%)$, no corno direito.

SCHRAMM ${ }^{18}$ verificou que, em 1.213 casos de gestaçర̄es diagnosticadas por palpação retal, em animais da raça Holandesa preta e branca, $479(39,49 \%)$ eram no corno uterino esquerdo e $734(60,50 \%)$ no como uterino direito, assinalando que no bovino a atividade dos ovários é maior no ovário direito $(60,00 \%)$ do que no ovário esquerdo $(40,00 \%)$. Em 521 casos onde o sexo do produto foi anotado, $195(37,43 \%)$ gestaçס̄es ocorreram no corno uterino esquerdo: 97 machos $(49,74 \%)$ e 98 fêmeas $(50,26 \%)$. No corno uterino direito ocorreram $326(62,57 \%)$ gestaçoes, sendo 166 de machos $(50,92 \%)$ e 160 de fêmeas $(49,08 \%)$. Concluiu o autor pela nāo relação entre o sexo do bezerro e o como gestante.

STALFORS ${ }^{19}$ verificou por palpaçāo retal em 923 gestaçōes, que $577(62,50 \%)$ foram no corno uterino direito e $346(37,50 \%)$ no corno uterino esquerdo.

ERDHEIM $^{5}$, examinando 3.824 úteros obtidos em matadouro, verificou em um primeiro estudo que 1.171 órgãos apresentavam gestaçăo simples, dos quais 644 foram de vacas leiteiras e 527 de vacas de corte; das primeiras, 455 $(69,10 \%)$ gestaçōes estavam no corno uterino direito e 199 $(30,90 \%)$ no corno uterino esquerdo; nas segundas, 262 $(49,72 \%)$ estavam no corno uterino direito e 265 $(50,28 \%)$ no corno uterino esquerdo. Em um segundo estudo, 2.617 úteros que também apresentavam gestaçðes simples, 845 foram de vacas leiteiras e 1.772 de vacas de corte. Nas primeiras, as gestaçðes se localizavam, $570(67,46 \%)$ no como uterino direito e $275(32,54 \%)$ no como uterino esquerdo; nas segundas $916(51,69 \%)$ no como uterino direito e $856(48,31 \%)$ no como uterino esquerdo.

NEILSEN 12 comunicou que de 5.970 vacas examinadas para diagnóstico de gestação, $59,20 \%$ apresentavam prenhez no corno uterino direito e $40,80 \%$ no corno uterino esquerdo. Em 4.290 novilhas, esta proporçáo foi de $54,40 \%$ e $45,60 \%$, respectivamente.

PERKINS et alii ${ }^{13}$, estudando 255 animais prenhes abatidos, encontrou $57,30 \%$ em gestaçāo no como uterino direito e $42,70 \%$ no corno uterino esquerdo, concordando suas observações com a dos autores por ele consultados que encontraram mais atividade do ovário direito em relaçăo 
ao ovário esquerdo.

VARENIKA $^{20}$ realizou 1.520 palpaç̄̃es retais em 175 animais, verificando que $60,90 \%$ das ovulações ocorreram no ovário direito, $33,90 \%$ no ovário esquerdo e $1,10 \%$ em ambos os ovários; nasceram 57 machos e 42 fêmeas gestadas no corno uterino direito e 44 machos e 32 fêmeas gestadas no corno uterino esquerdo. Por esses dados verifica-se que $56,57 \%$ foram gestados no como direito, sendo $57,58 \%$ machos e $42,42 \%$ de fêmeas e $43,43 \%$ foram gestados no corno esquerdo, $57,90 \%$ de machos e $42,10 \%$ de fêmeas.

FOOTE et alii ${ }^{7}$, realizando 536 diagnósticos de gestação por palpação retal em vacas holandesas variedade malhada de preto, encontraram 303 (56,53\%) gestantes no corno uterino direito (149 machos, $49,17 \%$ e 154 fêmeas, $50,83 \%)$ e $233(43,47 \%)$ no corno uterino esquerdo (103 machos, $44,21 \%$ e 130 fêmeas, $57,97 \%$ ).

FOOTE et alii 6 , trabalhando com 128 gestaçōes de 48 fêmeas da raça Angus e com 72 gestaçōes de 35 fêmeas da raça Shorthorn, encontraram 78 gestações no corno uterino direito $(60,94 \%)$ e 50 gestações no corno uterino esquerdo $(39,06 \%)$ para a primeira raça e 44 gestaçб̃es no corno direito $(61,11 \%)$ e 28 gestaçбes no corno esquerdo $(38,89 \%)$ para a segunda raça.

Em 133 úteros de animais prenhes da raça Highland sueca, SETTERGREN \& GALLOWAY ${ }^{17}$ acharam 59,40\% de gestaçסes no lado direito e $40,60 \%$ no esquerdo. Esta série inclúa um exemplar no qual o corpo lúteo estava localizado no ovário esquerdo e o feto no como direito.

GUERREIRO $^{9}$, em 1.960 gestaçס̄es simples em fêmeas da raça Holandesa, encontrou 1.220 no como uterino direito $(62,24 \%)$ com 694 machos $(56,88 \%)$ e 526 fêmeas $(43,12 \%) ; 740$ gestações no como uterino esquerdo $(37,76 \%)$ com 422 machos $(57,03 \%)$ e 318 fêmeas $(42,97 \%)$; ainda, na análise de 1.962 gestações, o autor encontrou $62,18 \%$ de gestaçठes que ocorreram no corno uterino direito e $37,81 \%$ no corno uterino esquerdo. Concluiu, também, que não existe qualquer relação entre o sexo do feto e o lado que ocupa no útero, evidenciando uma vez mais que a maioria das gestaçōes ocorreram no corno uterino direito em relaçáo ao esquerdo.

BEN-DAVD ${ }^{3}$ estudou 1.274 novilhas e 2.550 vacas. As novilhas apresentaram $1.113(87,40 \%)$ das gestações no como uterino direito e $161(12,60 \%)$ no corno uterino esquerdo. As vacas $2.246(88,00 \%)$ gestaram no como uterino direito e $304(12,00 \%)$ gestaram no corno uterino esquerdo.

AL-DAHASH \& DAVID ${ }^{1}$, analisando em matadouro 1.846 úteros com prenhez simples, verificaram que 1.136 $(61,54 \%)$ fetos se localizavam no corno uterino direito e $710(38,46 \%)$ estavam no como uterino esquerdo.

Examinando 287 vacas nativas prenhes abatidas, c^NTOS \& GILO ${ }^{16}$ encontraram $66,20 \%$ gestaçסes no corno uterino direito e $33,80 \%$ gestantes no como uterino esquerdo.

A localização dos sintomas de gestação nos cornos u- terinos direito e esquerdo, verificando-se a altemância da gestação e um possível efeito do corno prenhe sobre o sexo do produtojé o objetivo do presente estudo.

\section{MATERIAL E MÉTODOS}

No período de 1963 a 1979 foram examinadas 1.275 fémeas bovinas de exploraçáo leiteira, pertencentes a vários rebanhos localizados nas regiōes de Campinas, Sorocaba e Vale do Paraíba, no estado de São Paulo. Os animais eram da raça Holandesa, variedades malhada de preto e maihada de vermelho, considerados de elevado padrão zootécnico, puros de origem e puros por cruzamento e registrados nas respectivas Associaçбes de classe. Os rebanhos foram mantidos em regime de semi-estabulação e submetidos à monta natural e d inseminaçăo artificial.

Para obtenção dos dados, fêmeas normais de várias $\mathrm{i}$ dades, em reprodução, foram examinadas através da palpaçฐo retal, para verificaçăo das estruturas intemas do aparelho genital. Nestas condiçoes, dedicou-se atenção especial em examinar, através do tato, os cornos uterinos direito e esquerdo.

Para o diagnóstico da prenhez e do corno gestante, seguiu-se a técnica descrita por $\mathrm{HANCOCK}{ }^{11}$. Os exames foram efetuados entre 45 (quarenta e cinco) e 90 (noventa) dias após o serviço, nas fêmeas que não retornaram ao estro naquele período. Durante os trabalhos para obtenção do diagnóstico, os seguintes sintomas foram observados:

a. assimetria entre os cornos uterinos;

b. presença de flutuação ao longo do corno uterino, dada pelos fluídos do feto;

c. presença do feto;

d. presença de envoltórios fetais pelo "teste da parede dupla".

A prenhez positiva de corno uterino direito ou esquerdo era anotada ao lado da data do serviço, pelas siglas PCD e PCE, respectivamente. Quando do nascimento do bezerro, o sexo do mesmo era verificado e anotado em seguida à data do diagnóstico de gestação.

De todos os partos controlados, 1.009 foram simples, os quais foram utilizados para a realizaçđo deste trabalho.

Constituiram-se grupos de fêmeas no corno uterino direito e esquerdo, procurando-se verificar a frequência de alternância de gestação entre os dois comos uterinos. Paralelamente, verificou-se o efeito do corno gestante sobre o sexo dos bezerros.

A análise estatística dos dados consistiu na aplicação do teste de qui-quadrado $\left(\mathrm{X}^{2}\right)$, segundo GOLDSTEIN ${ }^{8}$, tendo sido estabelecido o nível de $5 \%$ para rejeição ou não da hipótese de nulidade (Ho).

\section{RESULTADOS}

Os resultados obtidos, bem como a análise estat ística dos dados, estáo contidos nas Tab. 1 e 2 . 
TABELA 1 - Frequencia e \% da gestação segundo cornos uterinos e raça, em fêmeas bovinas de exploração leiteira (1963 a 1979), São Paulo, 1982.

\begin{tabular}{|c|c|c|c|c|c|c|c|}
\hline \multirow[b]{2}{*}{ RAÇA } & & \multicolumn{2}{|c|}{ DIREITO } & \multicolumn{2}{|c|}{ ESQUERDO } & \multirow{2}{*}{ Total } & \multirow{2}{*}{$x^{2}$} \\
\hline & & $\mathrm{f}$ & $\%$ & $\mathrm{f}$ & $\%$ & & \\
\hline HOLANDESA & PO & 127 & 59,10 & 88 & 40,90 & 215 & $7,07 *$ \\
\hline $\begin{array}{l}\text { Malhada de } \\
\text { Preto }\end{array}$ & $\mathrm{PC}$ & 354 & 64,60 & 194 & 35,40 & 548 & $46,72 *$ \\
\hline HOLANDESA & PO & 55 & 58,50 & 39 & 41,50 & 94 & 2,72 \\
\hline $\begin{array}{l}\text { Malhada de } \\
\text { Vermelho }\end{array}$ & $\mathrm{PC}$ & 83 & 54,60 & 69 & 45,40 & 152 & 1,29 \\
\hline TOTAL & & 619 & 61,40 & 390 & 38,60 & 1.009 & - \\
\hline
\end{tabular}

$\mathrm{X}^{2}{ }_{5 \%}=3,84$ (1 G.L.)

P.O. = pura de origem

P.C. = pura por cruzamento

* = significante ao nivel de rejeição adotado

TABELA 2 - Efeito do corno uterino gestante sobre o sexo dos bezerros de fêmeas bovinas de exploração leiteira (1963 a 1979), São Paulo, 1982.

\begin{tabular}{|c|c|c|c|c|c|c|c|c|c|}
\hline \multirow{2}{*}{\multicolumn{2}{|c|}{ RAÇA }} & \multicolumn{4}{|c|}{ DIREITO } & \multicolumn{3}{|c|}{ ESQUERDO } & \multirow[b]{2}{*}{$x^{2}$} \\
\hline & & Machos & Fêmeas & Total & $x^{2}$ & Machos & Fêmeas & total & \\
\hline HOLANDESA & PO & 56 & 71 & 127 & 1,77 & 38 & 50 & 83 & 1,64 \\
\hline $\begin{array}{l}\text { Malhada de } \\
\text { Preto }\end{array}$ & $\mathrm{PC}$ & 171 & 183 & 354 & 0,41 & 107 & 87 & 194 & 2,06 \\
\hline HOLANDESA & PO & 32 & 32 & 55 & 0,47 & 22 & 17 & 39 & 0,65 \\
\hline $\begin{array}{l}\text { Malhada de } \\
\text { Vermelha }\end{array}$ & $\mathrm{PC}$ & 47 & 36 & 83 & 1,46 & 42 & 27 & 69 & 3,26 \\
\hline TOTAL & & 306 & 313 & 619 & & 209 & 181 & 390 & - \\
\hline
\end{tabular}

$X^{\llcorner} 5 \%=3,84$ (1 G.L.)

P.O. = pura de origem

P.C. = pura por cruzamento 
Ao nível de $5 \%$ de rejeição, pode-se inferir que a frequência de gestaçōes no como uterino direito é significantemente maior do que a frequência de gestações no corno uterino esquerdo, quando se analisaram os dados da raça Holandesa variedade malhada de preto, puro por cruzamento. Nas fêmeas da mesma raça, puras de origem, a frequência foi significativa. $\mathrm{O}$ agrupamento dos bovinos da raça Holandesa, variedade malhada de vermelho, não revelou significancia para o teste de frequencia aplicado.

A influência do corno uterino sobre o sexo dos bezerros mostrou desvios năo significativos, conforme os valores observados na Tab. 2.

\section{DISCUSSÃO}

Os resultados observados na Tab. 1 demonstram, para os animais da raça Halandesa variedade malhada de preto, puros de origem, $59,10 \%$ das gestaçoes no corno uterino direito e $40,90 \%$ ocorrendo no corno uterino esquerdo; para os animais puros por cruzamento da mesma raça, foram encontradas $64,60 \%$ de gestaçōes no como uterino direito e $35,40 \%$ de gestaçoes no corno uterino esquerdo. Os valores encontrados, ao redor de $60,00 \%$ para o como uterino direito e $40,00 \%$ para o como uterino esquerdo, aproximam-se daqueles apontados por $\mathrm{CLARK}^{4}$, SCHRAMM ${ }^{18}$, ERHEIM $^{5}$, FOOTE et alii $^{7}$, GUERREIRO ${ }^{9}$, para vacas leiteiras; FOOTE et alii6, para vacas de corte, SETTERGREN \& GALLOWAY ${ }^{17}$, nas vacas de exploração mista; STALFORS ${ }^{19}$, NEILSEN ${ }^{12}$, PERKINS et alii ${ }^{13}$, VARENIKA ${ }^{20}, A L-D A H A S H ~ \&$ DAVI $^{1}$, SANTOS \& GILO ${ }^{16}$, para fêmeas da espécies bovina sem exploração econômica definida.

Essas observaçōes são significantes ao nivel de $5 \%$ pela aplicaçáo do teste de $\mathrm{X}^{2}$ aos nossos dados. Entretanto, são bastante diferentes daqueles de BEN-DAVID ${ }^{3}$ que encontrou uma percentagem de cerca de $88,00 \%$ de gestações para o como uterino direito e ao redor de $12,00 \%$ para o corno uterino esquerdo.

No lote de animais ora em discussão, existiu uma atividade ovariana direita maior do que a esquerda, confirmando assim as citaçoes de SALISBURY \& VANDEMARK ${ }^{15}$, ROBERTS $^{14}$, ARTHUR ${ }^{2}$, HAFEZ ${ }^{10}$.

Ainda na Tab. 1 no tocante à raça Halandesa variedade malhada de vermelho, pura de origem, encontramos $58,50 \%$ das gestaçбes no corno uterino direito e $41,50 \%$ ocorrendo no corno uterino esquerdo; as fêmeas puras por cruzamento apresentaram $54,60 \%$ de gestaçóes no como uteino direito e $45,40 \%$ no como uterino esquerdo.

Esses dados, quando submetidos ao teste de $\mathrm{X}^{2}$ não revelaram significância estatística ao nível de $5 \%$, contrariando os dados disponiveis na literatura (CLARK ${ }^{4}$, SCHRAMM ${ }^{18}$, ERDHEIM ${ }^{5}$, FOOTE et alii ${ }^{7}$, GUERREIRO $^{9}$, para vacas leiteiras; FOOTE et alii 6 , para vacas de corte; SETTERGREN \& GALLOWAY ${ }^{17}$, nas va- cas de exploração mista; STALFORS ${ }^{19}$, NEILSEN 12 PERKINS et alii 13 , VARENIKA ${ }^{20}$, AL-DAHASH d DAVID $^{1}$, SANTOS \& GILO ${ }^{16}$, para fêmeas da espécie bo vina sem exploração econômica definida), indicando que a maioria das gestaçōes ocorre no corno uterino direito. Des te modo, os valores encontrados para a raça Holandesa va riedade malhada de vermelho, mostram que houve uma i gualdade funcional para os ovários direito e esquerdo, con cordando com os enunciados por ERDHEIM ${ }^{5}$ para vaca: de corte, onde o ovário esquerdo mostra a mesma atividadł funcional que o ovário direito. Diferem por outro lado, da: afirmaçōes de SALISBURY \& VANDERMARK ${ }^{15}$ ROBERTS $^{14}$, ARTHUR ${ }^{2}$, HAFEZ ${ }^{10}$, que afirmam que $\mathrm{nc}$ gado bovino, $60,00 \%$ da ovulaçōes ocorrem no ovário direi to e $40,00 \%$ no esquerdo.

CLARK $^{4}$, VARENIKA ${ }^{20}$, FOOTE et alii ${ }^{7}$, mostran resultados sobre a percentagem de machos e de fêmeas que nasceram do como uterino direito e do corno uterino es querdo, mas não concluem se existe ou não correlação entrє o sexo do bezerro e o corno uterino onde o mesmo foi gera do.

Os valores apresentados na Tab. 2, relativos ao efeitc do corno uterino gestante sobre o sexo do bezerro de fêmeas bovinas de exploração leiteira, quando analisados pelo teste de $\mathrm{X}^{2}$, não mostraram significância estat ística ao nivel de 5\%. Esses resultados confirmam as observaçōes de SCHRAMM $^{18}$ e de GUERREIRO ${ }^{9}$, de que na verdade, o sexo do bezerro não apresenta relaçāo com o como uterino em que foi gerado.

\section{CONCLUSOES}

Pelos resultados obtidos conclui-se:

- nas fêmeas bovinas da raça Holandesa variedade malhada de preto, puras de origem e puras por cruzamento, existe maior frequência de gestaçסes no corno uterino direito em relação ao corno uterino esquerdo ao nível de $5 \%$ de significância pelo teste $\mathrm{X}^{2}$;

- nas fêmeas bovinas da raça Holandesa variedade malhada de vermelho, tanto puras de origem como puras por cruzamento, não existe diferença estatisticamente significante ao nível de $5 \%$ pelo teste de $\mathrm{X}^{2}$, para as frequências de gestaçðes entre o corno uterino direito e o corno uterino esquerdo;

- nas fêmeas bovinas da raça Holandesa, variedade malhada de preto e variedade malhada de vermelho, tanto r.-as ' jem como puras por cruzamento, não existe djferença estatisticamente significante ao nivel de $5 \%$ pelo teste de $\mathrm{X}^{2}$, entre o sexo do bezerro e o corno uterino onde o mesmo foi gerado. 
SILVEIRA, W.F.; REIS, J.M.; BIZUTTI, O.; GAMBINI, L.B.; SANTOS, C.O.M. Study of the incidence of righthom and left-horn pregnancies, a possible correlation between the uterine horn and the sex of the calves. Rev.Fac.Med.vet.Zootec.LinivS. Paulo, 22(1):59-64, 1985.

SLMMARY: Through retal palpation the pregnant uterine horn was determined. Thus 1009 single pregnancies of Holstein and red Holstein cows, pure breed and cross-breed, were analysed. Through $\mathrm{X}^{2}$ (chisquare) analysis, fixing the rejection level of nullity hypothesis at $5 \%(P \leqslant 0.05)$, differences between pregnant uterine horns were statistically significant. Values were greater in the right uterine than in the left uterine horn in pure breed and cross-breed Holstein cows, being this distribution note similar in the pure breed cross-beed red Holstein cows. Both in Holstein and red Holstein cows the correlations between the sex of the calves and the pregnant uterine horns were not statistically significant.

UNITERMS: Uterine horn ${ }^{+}$; Pregnancy diagnosis ${ }^{+} ;$Sex ratio ${ }^{+}$; Cows

\section{REFERENCIAS BIBLIOGRÁFICAS}

1- AL-DAHASH, S.Y.A. \& DAVD, J.S.E. The incidence of ovarian activity pregnancy and bovine genital abnormalities shown by abattoir survey. Vet. Rec., 101(15):296-9, 1977.

2- ARTHUR, G.H. Veterinary reproduction and obstetrics. London, Baillière \& Tindall, 1975. p.70.

3- BEN-DAVID, B. Observations on the incidence of pregnancy in the left uterine hom in dairy cows. Refuah vet., 22(3): 183-7, 1965.

4- CLARK, C.F. Does the right ovary of the bovine function more frequently than the left? J. Amer. vet. med. Ass., 88(1):62-5, 1936.

5- ERHEIM, M. The incidence of right and left hom pregnancies in dairy and beef cattle. J. Amer. vet. med. Ass., 100(781): 3434, 1942.

6- FOOTE, W.D.; HAUSER, E.R.; CASIDA, L.E. Effect of uterine hom pregnant, parity of dam and sex of calf on birth weight and gestation lenght in Angus and Shorthorn cows. J. Anim. Sci., 19(2):470-3, 1960.

7- FOOTE, W.D.; TYLER, WJ.; CASIDA, L.E. Effect of some genetic and maternal environmental variations on birth weight and gestation lenght in Holstein cattle. J. Dairy Sci., 42(2): 305-11, 1959.

8- GOLDSTEIN, A. Biostatistics: an introductory test. 2. ed. New York, MacMillan, 1965.

9- GUERREIRO, R.T.C. Distribuição do lado da gestaf̧đo nas vacas holando-portuguesas. Variaçóes in- dividuais em sucessivas gestações. Rev. port. Ci. vet., 60(396):293-6, 1965.

10- HAFEZ, E.S.E., ed. Reprodução animal. Trad. por Renato Campanarut Bamabe. 4.ed. São Paulo, Manole, 1982. p.390.

11- HANCOCK, J.L. The clinical features of the reproductive organs of pregnant and non-pregnant cattle. Vet. Rec., 74(23): 646-52, 1962.

12- NEILSEN, F. Sterility in cattle specialy as a results of uterine infection. In: INTERNATIONAL VETERINARY CONGRESS, 14., London, 1949. v. 3 , sec. $4 c$, p.105.

13- PERKINS, J.R.; OLDS, D.; SEATH, D.M. A study of 1000 bovine genitalia. J. Dairy Sci., 37(10): $1158-63,1954$.

14- ROBERTS, S.J. Veterinary obstetrics and genital diseases. Ithaca, Edwards Brothers, 1971. p 377.

15- SALISBURY, G.W. \& VANDEMARK, N.L. Physiology of reproduction and artificial insemination of cattle. San Francisco, W.H. Freeman, 1961. p.70.

16- SANTOS, B.S. \& GILO, A. A study on the incidence of right-horn and left-horn pregnancies in slaughtered pregnant native cows and carabaos. Philipp. J. vet. Med., 11(1):75-80, 1972.

17- SETTERGREN, I. \& GALlOWAY, D.B. Studies on genital malformations in females cattles using slaughterhouse material. Nord. Vet. Med., 
17(1)-9-16, 1965.

18- SCHRAMM, W. Left sided and right sided pregnancy in cattle. Dtsch. tierarztl. Wschr., 45:387-9, 1937.

19- STALFORS apud REECE, RP. \& TERNER, C.W.
The functional activity of the right and left bovine ovary. J. Dairy Sci., 21(1):37-9, 1938.

20- VARENIKA, N. /Ovarian function in cows/ Vet Glas., Beograd, 11:649-54, 1957 apud Anim Breed. Abstr., 26(3):276, 1958.

Recebido para publicação em: 10/10/84

Aprovado para publicação em: 22/05/8 